\title{
Spleen tyrosine kinase promotes acute neutrophil- mediated glomerular injury via activation of JNK and p38 MAPK in rat nephrotoxic serum nephritis
}

\author{
Jessica Ryan ${ }^{1,2}$, Frank Y Ma ${ }^{1,2}$, John Kanellis ${ }^{1,2}$, Mercedes Delgado ${ }^{3}$, Kate Blease ${ }^{3}$ and David J Nikolic-Paterson ${ }^{1,2}$
}

Glomerular antibody deposition induces acute neutrophil-mediated glomerular injury via activation of c-Jun amino terminal kinase (JNK) and p38 mitogen-activated protein kinase (MAPK). However, the link between antibody deposition and activation of JNK/p38 MAPK signalling is unclear. This study tested the postulate that spleen tyrosine kinase (Syk), which is activated via Fc $\gamma$-receptor ligation, is required for activation of JNK and p38 signalling and acute neutrophil-mediated glomerular injury. We used a Syk inhibitor (SYKi) in rat nephrotoxic serum nephritis (NTN) in which neutrophil-mediated glomerular injury is dependent upon JNK and p38 signalling. SYKi or vehicle treatment of Sprague-Dawley rats began $30 \mathrm{~min}$ before administration of anti-GBM serum with rats killed 3 or $24 \mathrm{~h}$ later.

Immunostaining identified de novo glomerular Syk activation (p-Tyr 525/526) in untreated NTN, being most prominent in neutrophils. Vehicle and untreated NTN exhibited heavy proteinuria and glomerular thrombosis at $24 \mathrm{~h}$ with P-selectin and fibrin immunostaining within capillaries, glomerular macrophage and T cell infiltration, activation of JNK and p38 MAPK signalling, and upregulation of glomerular mRNA levels of pro-inflammatory molecules (TNF- $\alpha$, NOS2, MMP-12 and CCL2). In contrast, SYKi treatment provided complete protection from proteinuria, with a profound reduction in glomerular thrombosis and immunostaining for P-selectin and fibrin, and a substantial reduction in glomerular mRNA levels of pro-inflammatory molecules. SYKi treatment also reduced the acute glomerular neutrophil influx and pro-inflammatory response at $3 \mathrm{~h}$ in NTN. These protective effects were associated with a significant reduction in glomerular JNK and p38 MAPK activation. In addition, activation of Syk, JNK and p38 was identified in human biopsy samples of acute crescentic glomerulonephritis. In conclusion, this study demonstrates that Syk signalling is required for JNK and p38 MAPK signalling and acute neutrophil-dependent glomerular injury in rat NTN. These findings identify Syk as a potential therapeutic target in antibody-dependent kidney disease.

Laboratory Investigation (2011) 91, 1727-1738; doi:10.1038/labinvest.2011.137; published online 5 September 2011

KEYWORDS: glomerulonephritis; JNK; neutrophil; platelet; p38 MAPK; SYK

Studies of experimental kidney disease have established that glomerular antibody deposition can induce acute podocyte injury and proteinuria through Fc-receptor-dependent recruitment and activation of circulating neutrophils and monocytes. ${ }^{1-3}$ Leukocyte-mediated renal injury in antibodydependent models of kidney disease, such as nephrotoxic serum nephritis (NTN), operates via activation of the stressactivated protein kinases, c-Jun amino terminal kinase (JNK) and $\mathrm{p} 38$ mitogen-activated protein kinase (MAPK). ${ }^{4,5}$ However, we do not know the upstream components involved in activation of JNK and p38 MAPK pathways in this type of glomerular injury. One potential mechanism of
$\mathrm{JNK} / \mathrm{p} 38$ activation in antibody-dependent glomerular injury is through Fc-receptor activation.

Spleen tyrosine kinase (Syk) is a $72-\mathrm{kDa}$ non-receptor tyrosine kinase that has an important role in receptor signalling in cells of the haemotopoeitic system including B cells, neutrophils and monocyte/macrophages, but is largely absent in T cells. ${ }^{6}$ Syk is best known as a component of signalling via $B$ cell receptors and Fc-receptors. ${ }^{7-10}$ In these, a ligand-stimulated receptor associates with a transmembrane protein that contains an immunoreceptor tyrosine-based activation motif, which is then rapidly phosphorylated by SRC family kinases, leading to recruitment and activation of

\footnotetext{
${ }^{1}$ Department of Nephrology, Monash Medical Centre, Clayton, VIC, Australia; ${ }^{2}$ Monash University Department of Medicine, Monash Medical Centre, Clayton, VIC, Australia and ${ }^{3}$ Celgene, San Diego, CA, USA

Correspondence: Professor DJ Nikolic-Paterson, DPhil, Department of Nephrology, Monash Medical Centre, 246 Clayton Road, Clayton, Victoria 3168, Australia. E-mail: david.nikolic-paterson@monash.edu

Part of this work was presented at the Annual Meeting of the American Society of Nephrology, Denver, 2010.

Received 3 June 2011; revised 21 July 2011; accepted 26 July 2011
} 
Syk. Syk is activated by phosphorylation at Tyr 525/526 and then binds to members of the VAV and phospholipase $\mathrm{C} \gamma$ families, which in turn lead to activation of several signalling pathways, including JNK and p38 MAPK. ${ }^{11}$

Syk inhibitor drugs have produced positive results in clinical trials of rheumatoid arthritis and seasonal rhinitis. ${ }^{12-15}$ Preclinical studies using Syk inhibitor drugs have provided evidence that blockade of Syk protects against antibodydependent kidney disease murine lupus. ${ }^{16,17}$ In addition, a Syk inhibitor has been shown to suppress macrophage-mediated renal injury in a model of NTN in WKY rats, which lacks the heterologous phase of neutrophil-dependent glomerular injury. ${ }^{18}$ Despite these promising results, however, we have no information regarding Syk activation in kidney disease. Thus, three important questions remain unresolved. First, is Syk activated in antibody-dependent glomerular injury? Second, is Syk activation required for activation of JNK/p38 MAPK signalling in antibody-dependent glomerular injury? Third, is Syk activation required for antibody-dependent acute neutrophil-mediated glomerular injury? To address these questions, we used a Syk inhibitor (SYKi) in a model of NTN in Sprague-Dawley rats in which proteinuria in the first $24 \mathrm{~h}$ is neutrophil mediated. ${ }^{19}$

\section{MATERIALS AND METHODS Syk Inhibitor}

The SYKi was synthesised by Celgene. SYKi inhibits Syk enzyme activity with an $\mathrm{IC}_{50}$ of $30 \mathrm{nM}$ and has partial activity against Flt3 (66 nM), KDR (107 nM) and JAK2 (116 nM); but has no activity against a panel of 55 other kinases including p38, JNK, c-kit $(>3 \mu \mathrm{M})$, Lyn or Lck $(\sim 1 \mu \mathrm{M})$. This is similar to the specificity of the most widely reported Syk inhibitor, R406. ${ }^{20}$ SYKi inhibits IgM-induced TNF- $\alpha$ production by human macrophages and anti-IgM activation of human B cells (data not shown).

\section{Rat Model of NTN}

All animal experiments were approved by Monash Medical Centre Animal Ethics Committee. NTN was induced in inbred female Sprague-Dawley rats (150-200 g; Monash Animal Services, Melbourne) as described previously. ${ }^{41}$ Briefly, rats were immunised by subcutaneous injection of sheep IgG in Freund's complete adjuvant followed 5 days later (termed day 0 ) by intravenous injection of sheep anti-rat GBM serum. Animals were killed 3 or $24 \mathrm{~h}$ after administration of anti-GBM serum injection. SYKi was prepared
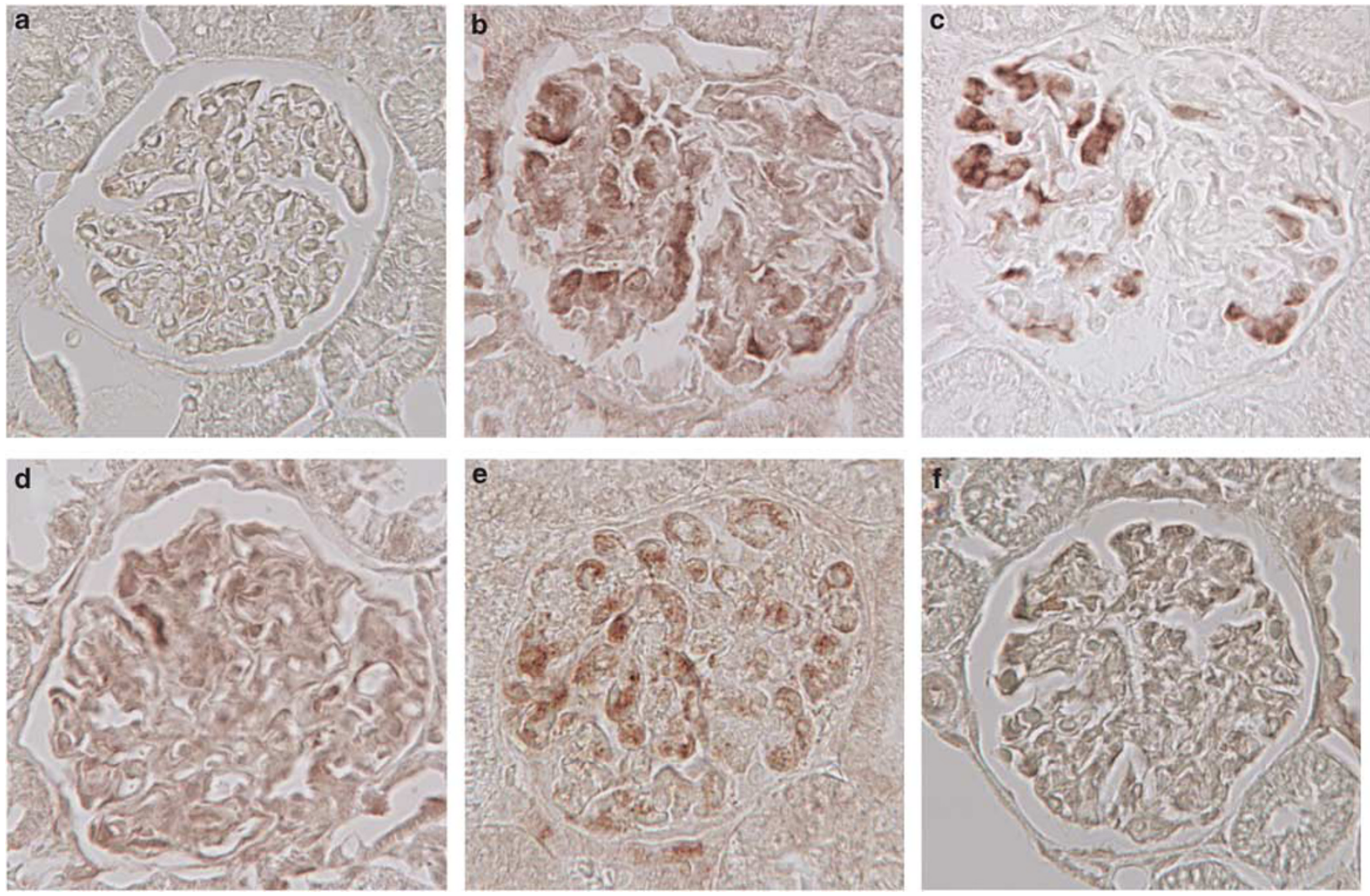

Figure 1 Immunostaining for p-Syk (Tyr 525/526) and RP-1 positive neutrophils in rat NTN. (a) Normal rat kidney lacks p-Syk staining. (b) Focal staining pattern of p-Syk staining in capillary loops in 3-h untreated NTN, which co-localises with (c) infiltrating RP1 + neutrophils. (d) In contrast, p-Syk staining is markedly reduced in NTN with SYKi treatment. (e) p-Syk staining is seen in capillary loops at $24 \mathrm{~h}$ in untreated NTN, and (f) this is abolished with SYKi treatment. 
as a suspension in $10 \%$ 2-hydropropyl- $\beta$-cyclodextrin (vehicle) and given by intraperitoneal injection, $10 \mathrm{mg} / \mathrm{kg}$, every $6 \mathrm{~h}$.

In the first experiment, groups of six or seven animals with SYKi treatment, vehicle treatment or no treatment were killed $24 \mathrm{~h}$ after administration of anti-GBM serum. SYKi treatment began $30 \mathrm{~min}$ before anti-GBM serum injection and continued until animals were killed at $24 \mathrm{~h}$. In addition, a group of normal rats was examined. Animals were housed in metabolic cages for $23 \mathrm{~h}$ after anti-GBM serum to collect urine. In the second experiment, groups of six animals were given SYKi or vehicle treatment $30 \mathrm{~min}$ before anti-GBM serum injection and animals were killed at $3 \mathrm{~h}$.

Blood was collected from animals at the time of death and white blood cell counts were performed on a Cell Dyn 3500 Cell Counter. Tissues from one kidney were either snap frozen, or fixed in $2 \%$ paraformaldehyde or $4 \%$ formalin and processed for histopathological analysis. One kidney from the animals in each group $(n=4-6)$ was used for glomerular isolation by differential sieving, with tubular fragments removed under microscopic examination. Urinary protein excretion was estimated using a Coomassie (Bradford) Protein Assay Kit (ThermoScientific, Rockford, USA).

\section{Renal Biopsies}

Formalin-fixed sections of kidney biopsy tissue was analysed from patients who had previously undergone diagnostic renal biopsy at Monash Medical Centre. Patients gave informed consent and this study was approved by the Monash Medical Centre Human Ethics and Research Committee. The four biopsies were of minimal change disease, thin membrane disease and two cases of post-infectious glomerulonephritis. None of the patients were on immunosuppressive therapy at the time of biopsy.

\section{Antibodies}

The following antibodies were used in this study: mouse antirat CD68 recognising monocytes/macrophages (ED1) and mouse anti-rat T-cell receptor (R73, Serotec, Oxford, UK); mouse anti-rat neutrophil (RP-1, Becton Dickinson, San Diego, CA, USA); rabbit anti-fibrinogen- $\gamma$, mouse antiP-selectin (CD62P) and rabbit anti-WT-1 (Santa Cruz Biotechnology, Santa Cruz, CA, USA); rabbit antibodies against phospho-Syk (Tyr 525/526), phospho-p38 (Thr 180/ Thr 182), phospho-JNK (Tyr183/Tyr185) (Cell Signalling, Danvers, MA, USA), rabbit anti-human neutrophil elastase (Abcam, Cambridge, MA) and; rabbit anti-tubulin (Abcam). Biotinylated secondary antibodies were goat anti-mouse IgG (Zymed, San Franciso, CA, USA) and goat anti-rabbit IgG (Invitrogen, Carlsbad, CA, USA), which were detected using a Vectastain ABC kit (Vector Laboratories, Burlingame, CA, USA). Other secondary detection systems included goat antirabbit Alexa Fluor 680 (Molecular Probes, Eugene, OR, USA), horseradish peroxidase-conjugated goat anti-rabbit IgG and goat anti-mouse IgG and mouse peroxidise-conjugated antiperoxidase complexes (PAP) (Dako, Glostrup, Denmark).

\section{Histology}

Periodic acid-Schiff staining was performed on $2 \mu \mathrm{m}$ sections of formalin-fixed tissue sections. A total of 30 glomeruli from each animal were examined under high power $(\times 400)$. The area of the glomerular tuft affected by capillary thrombi was assessed using a semi-quantitative score: 0 (Nil), $1(<25 \%)$, 2 (25 to $50 \%), 3(50$ to $75 \%)$ and $4(>75 \%)$. Analysis was performed on blinded slides.

\section{Immunohistochemistry}

Immunoperoxidase staining for fibrinogen- $\gamma$ and WT- 1 was performed on $4-\mu \mathrm{m}$ sections of formalin-fixed tissue sections using microwave-based antigen retrieval (citrate buffer, $\mathrm{pH}$ 6.0 or Tris-HCL, pH 8.5, respectively) followed by the Vectastain ABC method as previously described. ${ }^{21}$ Immunostaining for p-Syk, p-p38, p-JNK, CD68 and human neutrophils was performed on formalin-fixed sections with antigen retrieval (citrate buffer, pH 6.0). Immunostaining for rat neutrophils, $\mathrm{T}$ cells and P-selectin was performed on frozen sections using the PAP or ABC method.

The number of glomerular cells stained positive for RP1, R73, ED1, WT-1 and p-JNK were counted in at least 30 glomerular cross-sections from each animal under high power $(\times 400)$. Glomerular immunostaining for P-selectin, fibrinogen and p-p38 was assessed using a semi-quantitative score: 0 (Nil), 1 ( $<25 \%), 2$ ( 25 to $50 \%$ ), 3 (50 to $75 \%$ ) and 4 $(>75 \%)$. All analysis was performed on blinded slides.

\section{Real-Time PCR}

Real-time PCR was performed as described previously. ${ }^{22}$ RNA was extracted from isolated glomeruli and reverse transcribed using random primers to make cDNA (First-
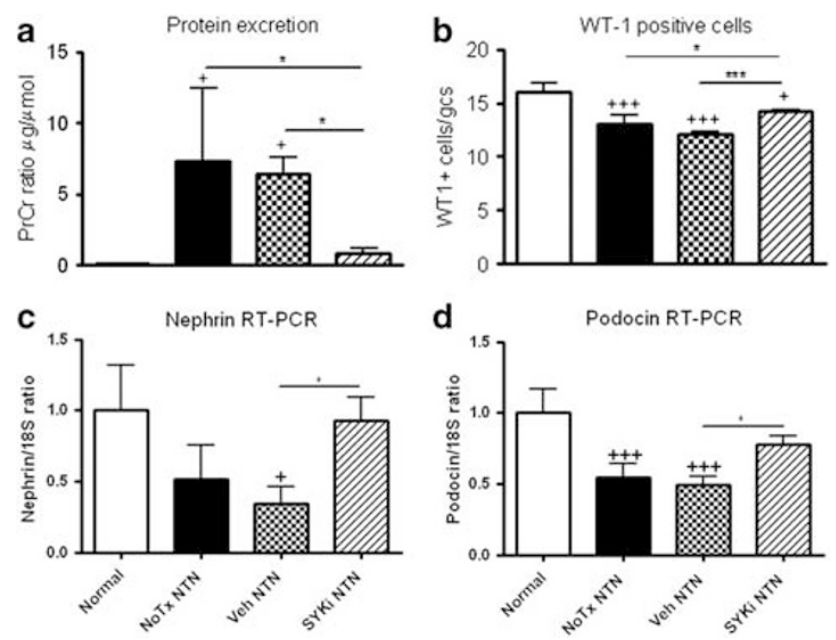

Figure 2 Renal injury in NTN. (a) Graph of urinary protein to creatinine ratio (PrCr in $\mu \mathrm{g} / \mu \mathrm{mol}$ ). (b) Graph showing the number of WT-1 + podocytes per glomerular cross-section (gcs). Real-time RT-PCR of isolated glomeruli showing relative mRNA levels for nephrin (c) and (d) podocin. ${ }^{+} P<0.05$, $+++P<0.001$ vs normal, ${ }^{*} P<0.05,{ }^{* * *} P<0.001$ vs SYKi. 
Strand Synthesis System for RT-PCR, Invitrogen). Real-time PCR was performed on a StepOne machine (Applied Biosystems, Scoresby, VIC, Australia) with thermal cycling conditions of $37^{\circ} \mathrm{C}$ for $10 \mathrm{~min}, 95^{\circ} \mathrm{C}$ for $5 \mathrm{~min}$, followed by 50 cycles of $95^{\circ} \mathrm{C}$ for $15 \mathrm{~s}, 60^{\circ} \mathrm{C}$ for $20 \mathrm{~s}$ and $68^{\circ} \mathrm{C}$ for $20 \mathrm{~s}$. The primer pairs and FAM-labeled MGB probes were as
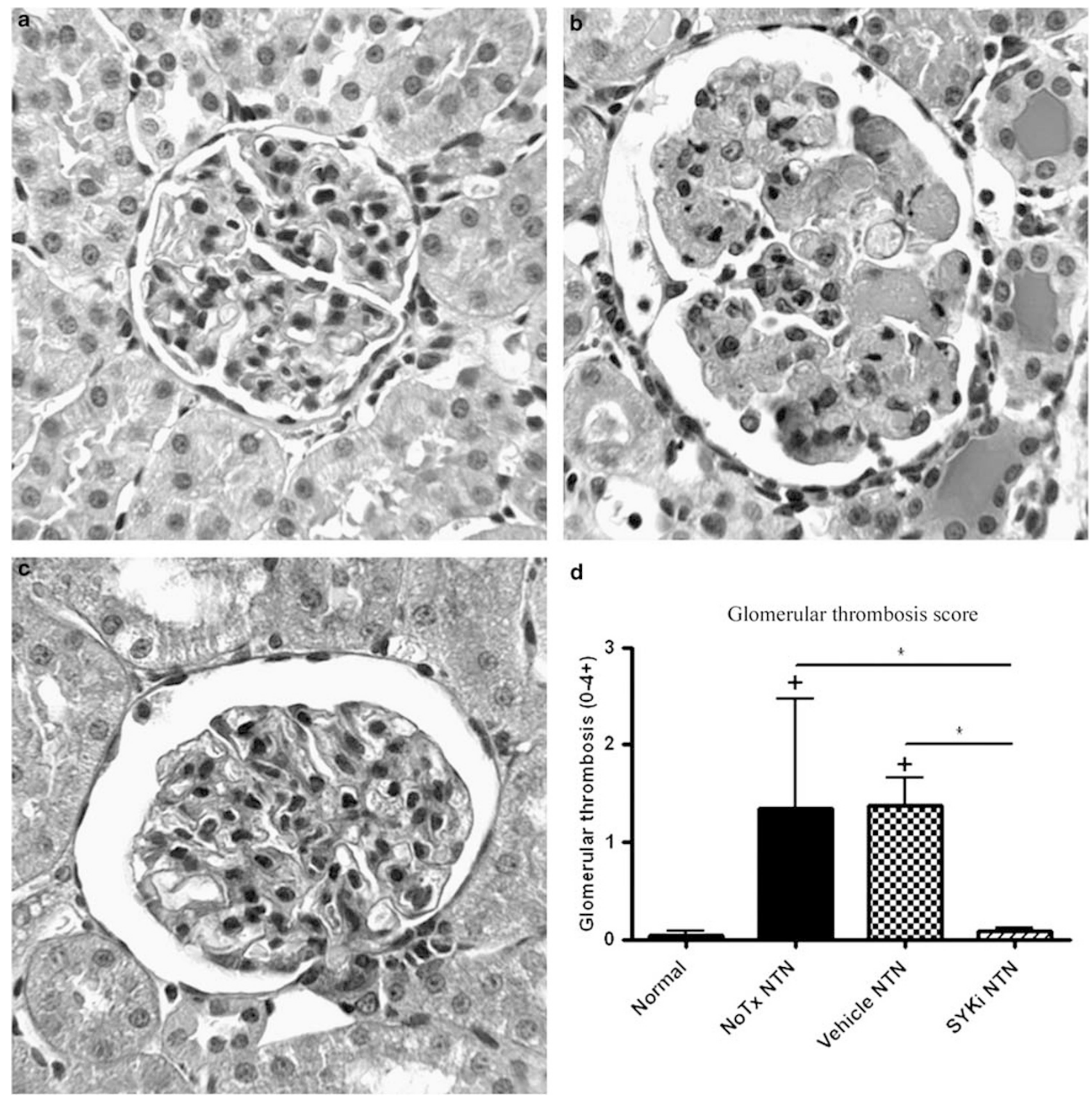

d

Glomerular thrombosis score

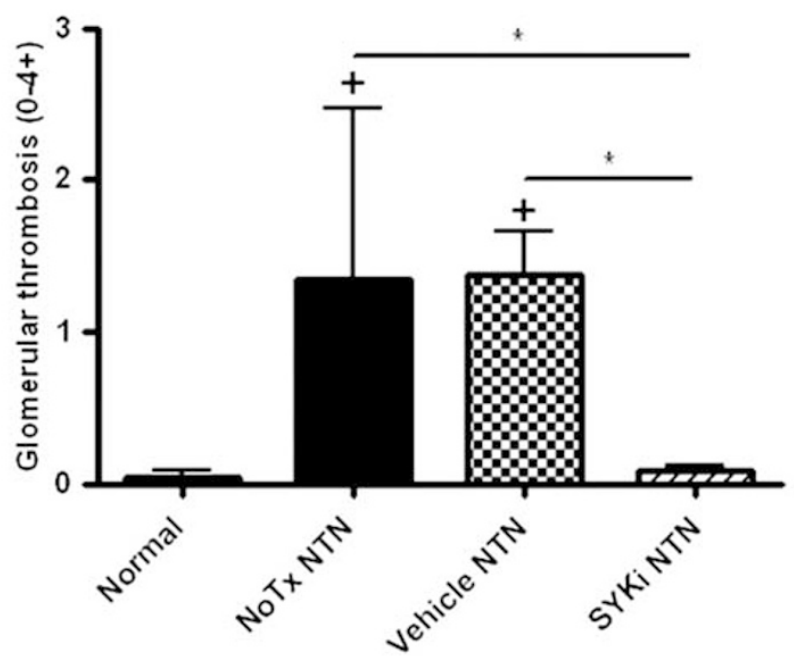

Figure 3 Renal damage assessed on PAS-stained sections. (a) Normal rat kidney. (b) Untreated 24-h NTN showing glomerular capillary thrombosis and tubular protein casts. (c) A $24 \mathrm{~h}$ NTN with SYKi treatment showing mild hypercellularity, but no thrombi. (d) Graph of glomerular thrombosis score (0-4 +). ${ }^{+} P<0.05$ vs normal, ${ }^{*} P<0.05$ vs SYKi.

Figure 4 Immunostaining for P-selectin and fibrinogen in rat NTN. Immunostaining for P-selectin in (a) normal rat kidney, (b) untreated 24-h NTN and (c) SYKi-treated 24-h NTN. (d) Graph showing semi-quantitative scoring of glomerular P-selectin staining. Immunostaining for fibrin deposition in (e) normal rat kidney, (f) untreated 24-h NTN and (g) SYKi-treated 24-h NTN. (h) Graph showing semi-quantitative scoring of glomerular fibrinogen staining. (a-c and e-g) Original magnification, $\times 400 .{ }^{+} P<0.05,{ }^{++} P<0.01$ and ${ }^{+++} P<0.001$ vs normal; ${ }^{\star} P<0.05,{ }^{* *} P<0.01,{ }^{* * *} P<0.001$ vs SYKi. 

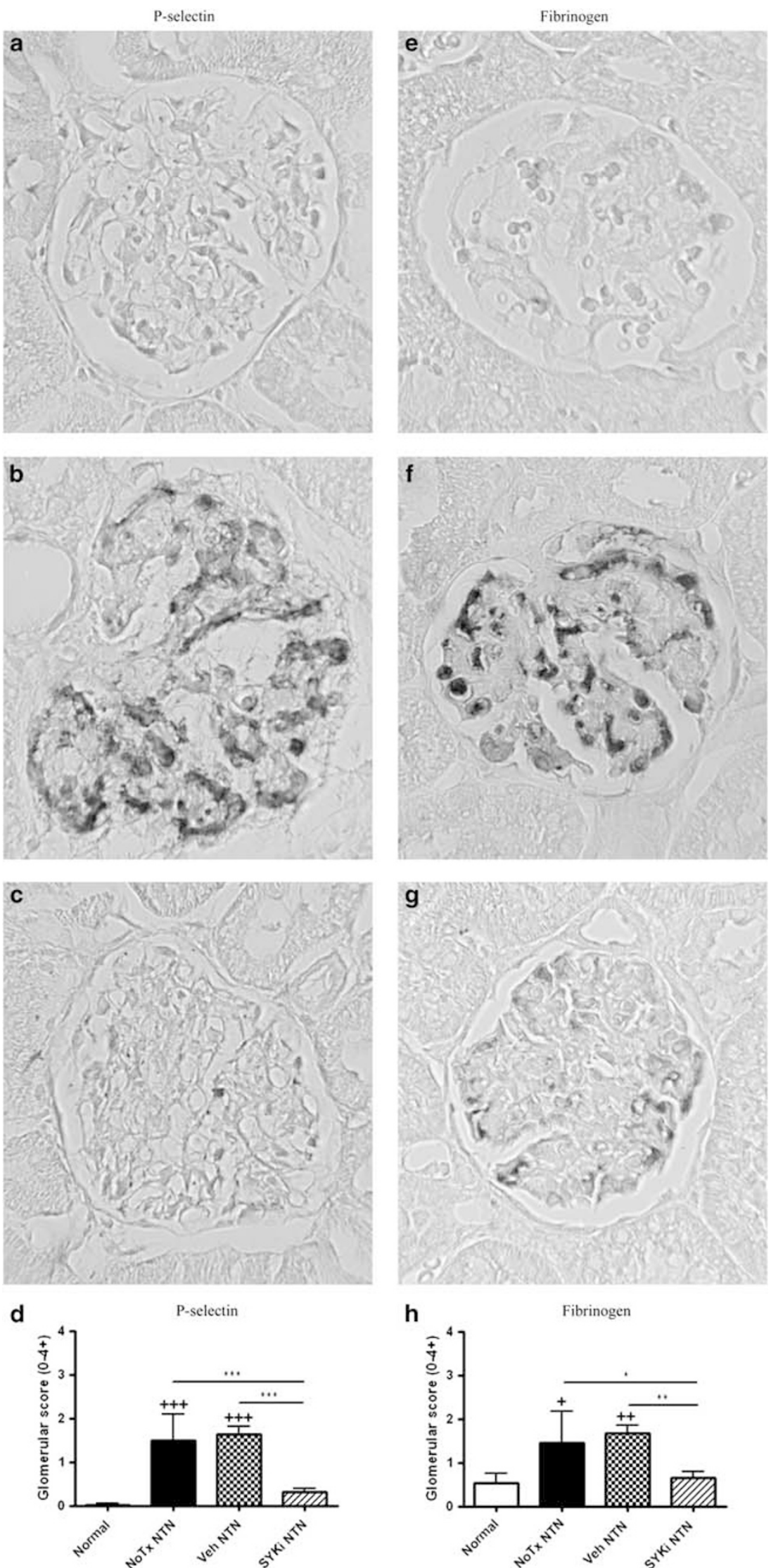
follows: nephrin (forward: 5'-CCATTACCATCCTGGGATC T-3'; reverse: 5'-CCATCCATGACTGTCTCATCT-3'; probe: $5^{\prime}$-TGACCCTTTGCTGCCT- $3^{\prime}$ ) and podocin (forward: $5^{\prime}$-CA TCAAAGTGGAGAGAACTGAA- ${ }^{\prime}$; reverse: $5^{\prime}$-ACAGAATC TCAGCCGCCAT- $3^{\prime}$; probe: $5^{\prime}$-CACAAAGACAGGCCAA- ${ }^{\prime}$ ). Primers and probes for NOS2, TNF- $\alpha$, MMP-12 and CCL2 have been described previously. ${ }^{22}$ The relative amount of mRNA was calculated using comparative $\mathrm{Ct}(\Delta \Delta \mathrm{Ct})$ method. All specific amplicons were normalised against $18 \mathrm{~S}$ RNA, which was amplified in the same reaction as an internal control using commercial assay reagents (Applied Biosystems).

\section{Western Blot}

Isolated glomeruli were lysed in $1 \mathrm{ml}$ of RIPA buffer, centrifuged and the supernatant was stored as described previously. ${ }^{23}$ Lysates were separated by SDS-PAGE using $12 \%$ acrylamide gels, electroblotted onto a PVDF membrane, and the blots were incubated for $1 \mathrm{~h}$ with Odyssey blocking buffer (Millennium Science, VIC, Australia). Blots then were incubated with the antibody of interest in blocking buffer overnight at $4{ }^{\circ} \mathrm{C}$. Blots were washed and then incubated with an Alexa Fluor-conjugated secondary antibody for $1 \mathrm{~h}$ at room temperature, washed and scanned using the Odyssey infrared Imaging System (Licor Biosciences). Membranes were re-probed with anti- $\alpha_{1}$ tubulin to determine equivalence of protein loading. Densitometry analysis was performed using the Gel Pro analyser Program (Media Cybernetics, Silver Spring, MD, USA).

\section{Statistics}

Data are presented as mean \pm s.d. Analysis between groups of animals was performed by parametric ANOVA using Bonferroni's post-test for multiple comparisons. Non-parametric data were analysed by the Kruskal-Wallis ANOVA by ranks using Dunn's post-test for multiple comparisons (GraphPad Prism 5.0 Software, San Diego, CA, USA).

\section{RESULTS}

\section{Syk is Activated in Rat NTN}

Activation of Syk occurs by autophosphorylation of Tyr 525/ 526 in the activation loop. ${ }^{24}$ Using immunohistochemistry, we identified p-Syk (Tyr 525/526) in glomerular cells at $3 \mathrm{~h}$ in vehicle-treated rat NTN, whereas staining was absent in normal glomeruli (Figures 1a and b). These p-Syk positive cells are mostly within capillary loops and the staining co-localised with infiltrating RP1 + neutrophils (Figures $1 \mathrm{~b}$ and c). Immunostaining for $\mathrm{p}$-Syk was also seen within capillary loops at $24 \mathrm{~h}$ in this model (Figure 1e). The glomerular p-Syk staining at $3 \mathrm{~h}$ and $24 \mathrm{~h}$ of NTN was largely abrogated by SYKi treatment (Figures $1 \mathrm{~d}$ and $\mathrm{f}$ ).

\section{SYKi Prevents Proteinuria}

All untreated and vehicle-treated animals developed proteinuria by $24 \mathrm{~h}$ of NTN (Figure 2a). The induction of proteinuria was associated with a reduction in the number of WT-1 + podocytes and downregulation of mRNA levels of the podocyte-specific molecules, nephrin and podocin (Figures $2 \mathrm{~b}-\mathrm{d}$ ). SYKi treatment prevented the development of proteinuria (Figure $2 \mathrm{a}$ ). SYKi treatment provided partial protection against podocyte loss, and SYKi prevented the reduction in mRNA levels of nephrin and podocin (Figures $2 \mathrm{~b}-\mathrm{d}$ ).

\section{SYKi Prevents Glomerular Thrombosis and Platelet Activation}

Glomerular injury was evident in vehicle and untreated NTN at $24 \mathrm{~h}$ as shown by glomerular hypercellularity and focal and segmental capillary thrombosis (Figures $3 \mathrm{a}$ and $\mathrm{b}$ ). There was relatively little tubulointerstitial damage apart from small numbers of protein casts. Platelet and endothelial activation in NTN was evident by the strong immunostaining for P-selectin and fibrinogen within glomerular capillary loops (Figure 4).

SYKi treatment gave profound protection from glomerular damage in rat NTN, with abrogation of capillary thrombosis and only mild glomerular hypercellularity evident (Figures $3 \mathrm{c}$ and d). SYKi treatment reduced immunostaining for P-selectin and fibrinogen by $81 \%$ and $61 \%$, respectively
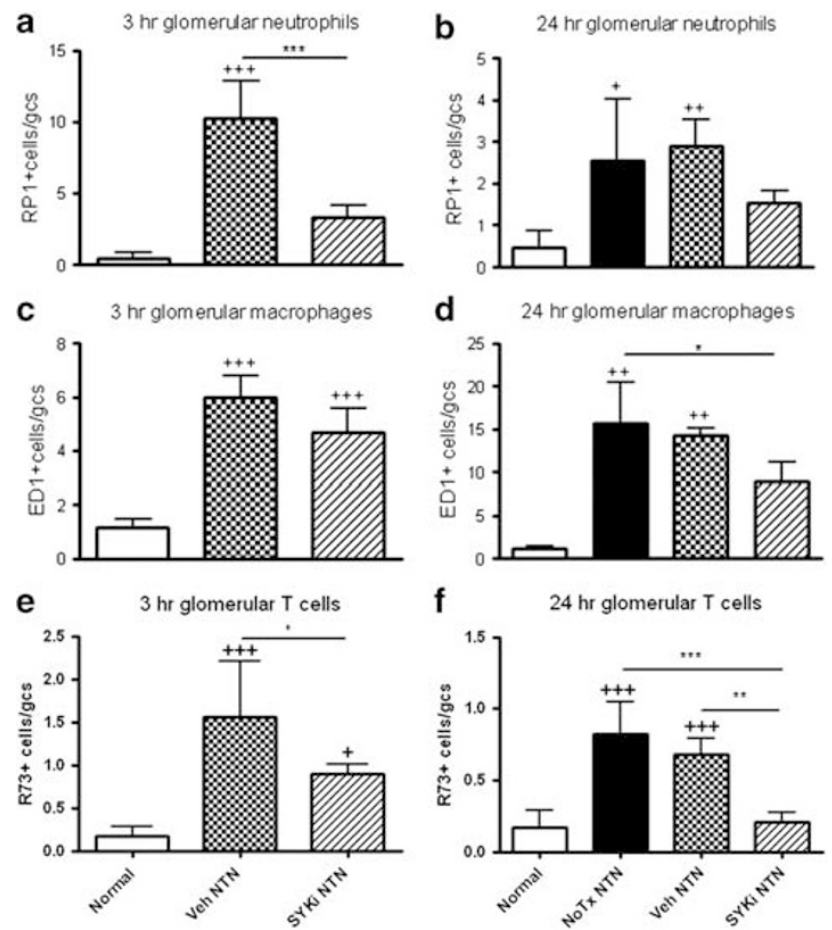

Figure 5 Quantification of glomerular leukocytes in NTN. (a-c) Graphs showing cell counts per glomerular cross-section (gcs) in normal rats and at $3 \mathrm{~h}$ NTN with vehicle or SYKi treatment for (a) RP1 + neutrophils, (b) ED1 + macrophages and (c) R73 + T cells. (d-f) Graphs showing cell counts in normal rats and at $24 \mathrm{~h}$ NTN with vehicle or SYKi treatment or no treatment (no Tx) for (d) RP1 + neutrophils, (c) ED1 + macrophages and (f) R73 + T cells. ${ }^{+} P<0.05,{ }^{++} P<0.01$ and ${ }^{+++} P<0.001$ vs normal; ${ }^{\star} P<0.05,{ }^{* *} P<0.01,{ }^{* * *} P<0.001$ vs SYKi. 
(Figures 4). Taken together, these data suggest that SYKi treatment significantly reduced both platelet and endothelial activation. Glomerular deposition of sheep IgG, rat IgG and rat $\mathrm{C} 3$ at $24 \mathrm{~h}$ was unaltered by SYKi compared with vehicle and untreated NTN (data not shown).

\section{SYKi Reduced Glomerular Leukocyte Recruitment}

Consistent with previous studies in this model of rat $\mathrm{NTN},{ }^{4,5,25}$ glomerular RP1 + neutrophil infiltration peaks $\sim 3 \mathrm{~h}$ after anti-GBM serum administration in untreated and vehicle animals and is largely resolved by $24 \mathrm{~h}$. Glomerular macrophage infiltrate is seen at $3 \mathrm{~h}$ and increases further at $24 \mathrm{~h}$, while the $\mathrm{T}$ cell infiltrate peaks at $3 \mathrm{~h}$ and is partially reduced by $24 \mathrm{~h}$ (Figure 5). SYKi treatment substantially reduced the peak of glomerular neutrophil influx at $3 \mathrm{~h}$ compared with vehicle-treated animals (Figure 5a). Glomerular $\mathrm{T}$ cell infiltration was reduced by SYKi treatment at 3 and $24 \mathrm{~h}$, while there was only a borderline effect upon the macrophage infiltrate (Figure 5). In addition, SYKi treatment did not affect white blood cell counts, which showed a trend towards neutrophilia in all groups with acute NTN (data not shown).

\section{SYKi Inhibited Activation of JNK and p38 MAPK}

We examined whether SYKi treatment modulates activation of the JNK and p38 MAPK pathways in rat NTN. Western blotting showed a significant increase in phosphorylation of JNK and p38 in isolated glomeruli in vehicle and untreated NTN, and this activation was prevented by SYKi treatment (Figure 6). Immunostaining identified a marked increase in the number of $\mathrm{p}$-JNK + cells and p-p38 + cells in glomeruli in vehicle and untreated NTN, and this response was essentially abrogated by SYKi treatment (Figure 7). These results demonstrate that SYKi treatment prevents activation of JNK and p38 MAPK pro-inflammatory pathways in rat NTN.

\section{SYKi Inhibits Glomerular Inflammation}

The inflammatory response in isolated glomeruli was examined by real-time RT-PCR. Vehicle and untreated animals showed a robust upregulation of TNF- $\alpha$, NOS2, MMP12 and CCL2 mRNA levels at 3 and $24 \mathrm{~h}$ of NTN. SYKi substantially reduced mRNA levels of all of these proinflammatory molecules at 3 and $24 \mathrm{~h}$ (Figure 8).

\section{Syk is Activated in Human Post-Infectious Glomerulonephritis}

We sought to determine the relevance of these experimental findings to human acute neutrophil-mediated glomerular injury. We examined two cases of post-infectious glomerulonephritis, a disease generally associated with glomerular IgG and IgM deposition and a neutrophil-rich leukocytic infiltrate. No p-Syk staining was seen in cases of minimal change and thin membrane disease, which were chosen to represent 'normal' kidney without antibody deposition or leukocyte infiltration (Figure 9a). In both cases of post-infectious glomerulonephritis, numerous p-Syk + cells were evident in glomeruli as illustrated in Figure 9b. Immunostaining of serial sections demonstrated that these glomerular p-Syk + cells co-localise with infiltrating neutrophils (Figures $9 \mathrm{c}$ and $\mathrm{d}$ ), showing a focal glomerular staining pattern similar to that seen in rat NTN (Figure 1). Furthermore, immunostaining for p-JNK and p-p38 followed a similar pattern to that of p-Syk in postinfectious glomerulonephritis (Figures 9e and f). a
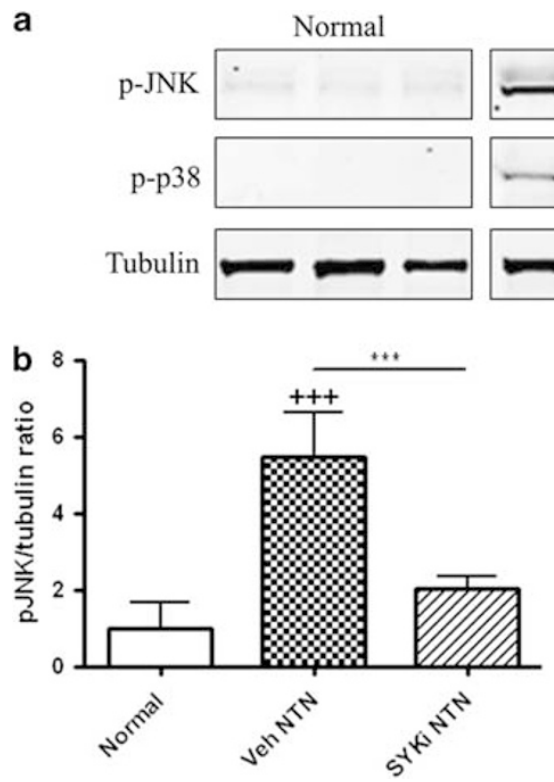

Vehicle
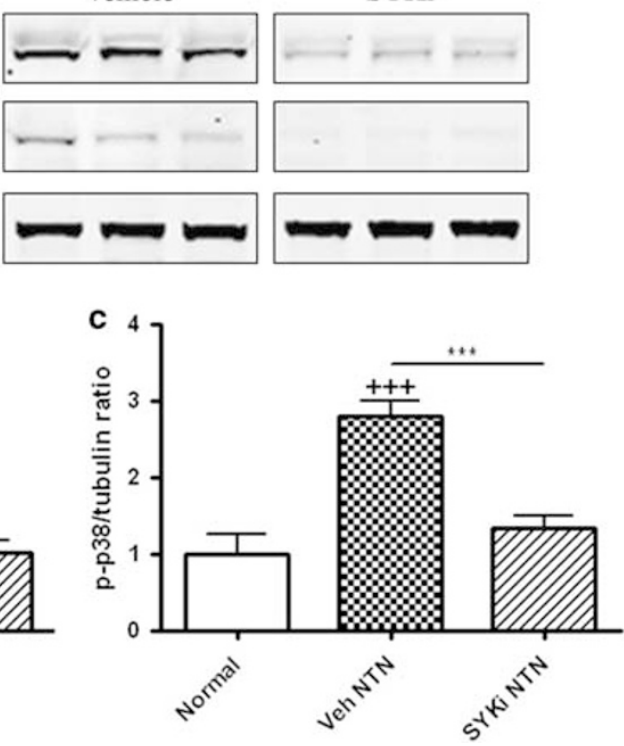

Figure 6 JNK and p38 activation in NTN. (a) Isolated glomeruli from normal rats, vehicle treated and SYKi treated 24-h NTN were analysed by western blotting for $\mathrm{p}-\mathrm{JNK}$ and $\mathrm{p}-\mathrm{p} 38$ and blots reprobed for tubulin. Graphs showing quantification of (b) $\mathrm{p}-\mathrm{JNK}$ and (c) $\mathrm{p}-\mathrm{p} 38$. ${ }^{+++} \mathrm{P}<0.001$ vs normal; ${ }^{* * * P}<0.001$ vs SYKi. 

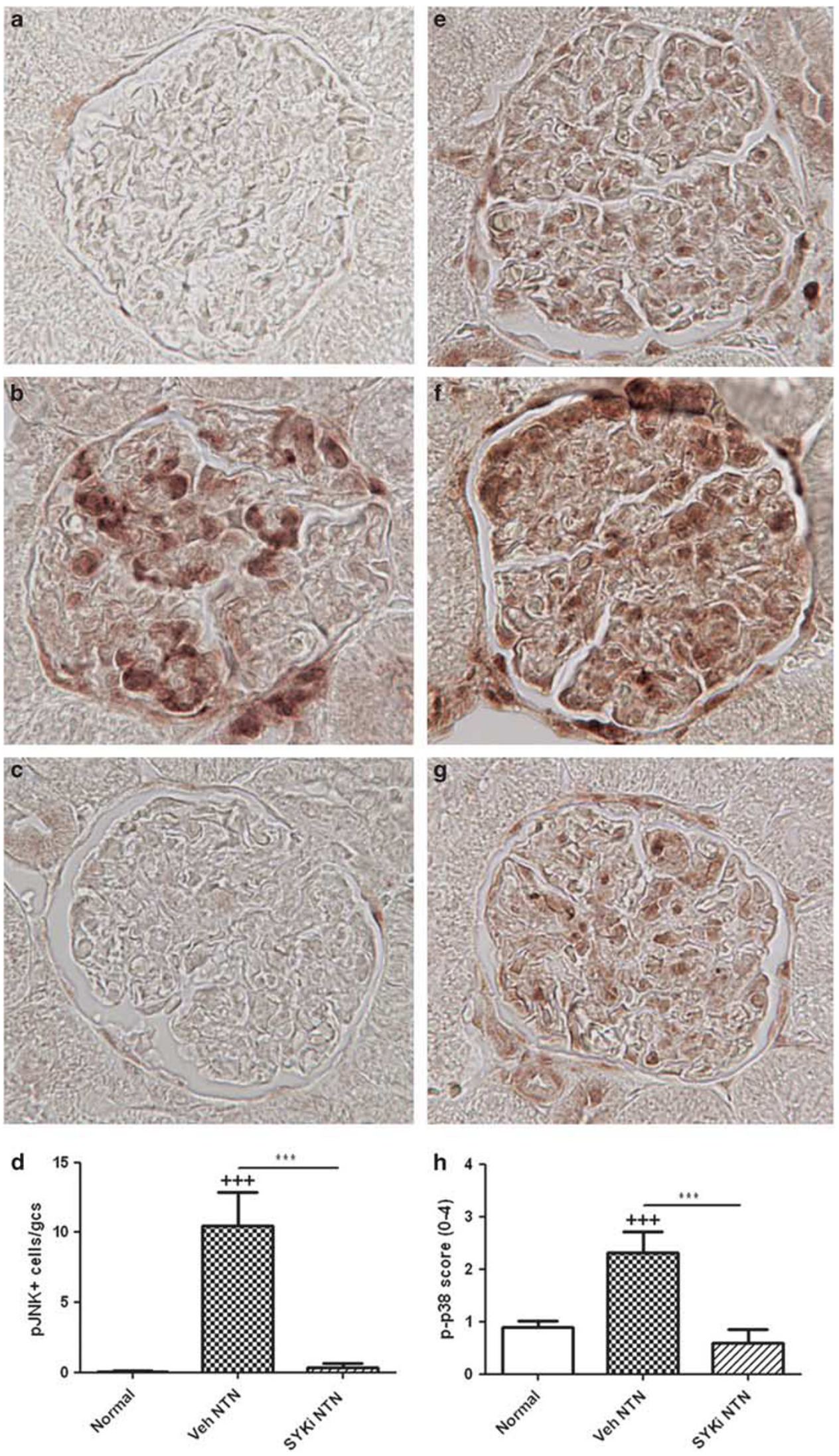


\section{DISCUSSION}

This study has three novel findings: detection of Syk activation in human and experimental kidney disease, demonstration that a Syk inhibitor drug can prevent acute neutrophil-mediated glomerular injury and identification of Syk as an important upstream activator of JNK and p38 signalling pathways in acute glomerulonephritis.

It is well established that Syk has an important role in Fc-R-mediated activation of neutrophils, monocytes and mast cells $;^{11}$ however, direct observation of Syk activation in vivo has been lacking. In this study, we have used
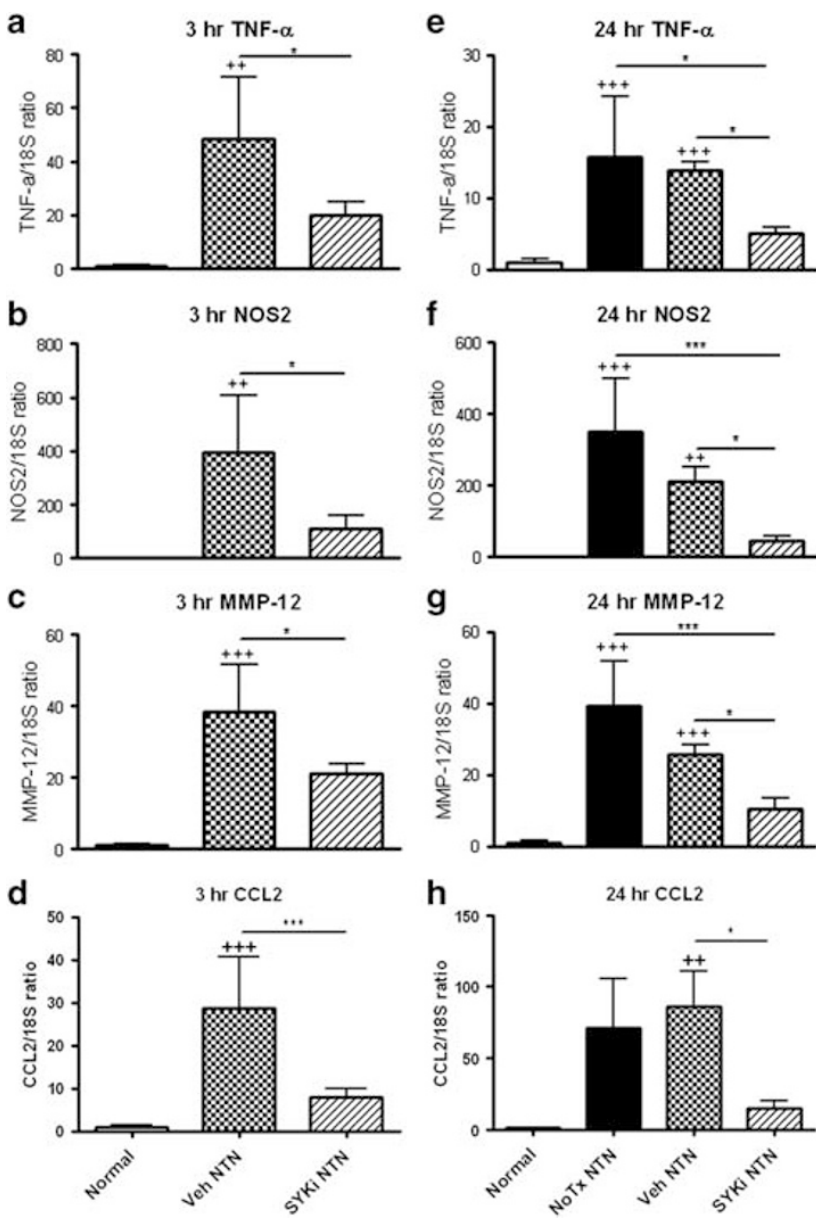

Figure 8 Real-time RT-PCR of isolated glomeruli from normal and NTN rats. (a-d) Analysis of relative mRNA levels in normal rats and in vehicle and SYKi-treated animals at $3 \mathrm{~h}$ NTN for (a) TNF- $\alpha$, (b) NOS2, (c) MMP-12 and (d) CCL2. (e-h) Analysis of relative mRNA levels in normal rats and in vehicletreated, SYKi-treated and untreated animals at $24 \mathrm{~h}$ NTN for (e) TNF- $\alpha$, (f) NOS2, (g) MMP-12 and (h) CCL2. ${ }^{++} P<0.01$ and ${ }^{++}+P<0.001$ vs normal; ${ }^{*} P<0.05$ and ${ }^{* *} P<0.001$ vs SYKi. immunohistochemistry to identify Syk activation on the basis of phosphorylation of its activation loop. Glomeruli in normal kidney showed no evidence of Syk activation, but p-Syk staining was evident within glomerular capillaries in acute NTN in a pattern suggesting activation in neutrophils, platelets and macrophages. Importantly, we also observed Syk activation in biopsies of post-infectious glomerulonephritis in a staining pattern, which co-localised with infiltrating neutrophils, whereas minimal change disease and thin membrane disease showed no evidence of Syk activation. These findings open the way for a detailed examination of Syk activation in human glomerular diseases.

It is well established that glomerular antibody deposition can induce acute renal injury via Fcy-receptor-dependent activation of platelets and neutrophils. ${ }^{2,3,19,26}$ Syk inhibitors have been shown to suppress macrophage-mediated renal injury, ${ }^{17,18,27}$ but whether Syk is also involved in neutrophildependent glomerular injury is unknown. In this study, blockade of Syk signalling using SYKi reduced podocyte damage and prevented proteinuria. There are several potential mechanisms, which may, individually or in combination, have contributed to this protective effect.

Firstly, SYKi treatment substantially reduced the peak of glomerular neutrophil influx at $3 \mathrm{~h}$, which is critical for induction of acute proteinuria in this disease model. ${ }^{19}$ This inhibition of neutrophil influx could be due to several mechanisms, although depletion of circulating neutrophils and platelets was ruled out. Platelets have an important role in glomerular neutrophil recruitment and the development of acute proteinuria, ${ }^{26,28}$ and blockade of P-selectin (CD62P) has been shown to prevent glomerular neutrophil recruitment and acute proteinuria. ${ }^{28,29}$ In our study, untreated and vehicle-treated NTN showed extensive glomerular capillary thrombi, together with P-selectin expression and fibrin deposition. SYKi treatment provided marked protection from histological damage, as well as abrogating glomerular P-selectin and fibrinogen staining. Thus, blockade of platelet activation and/or P-selectin expression are likely causes of reduced neutrophil influx. Indeed, a role for Syk has been described in signal transduction by the major P-selectin ligand expressed by leukocytes, P-selectin glycoprotein ligand $1 .^{30,31}$ Syk signalling also has an important role in platelet activation via the Fc-R $\gamma$ associated receptor, GP-VI. ${ }^{32}$ In addition, Syk signalling has been implicated in integrin signalling, both in platelet activation and in neutrophil adhesion. $^{33,34}$

Secondly, SYKi treatment suppressed glomerular inflammation at the $3 \mathrm{~h}$ time point as shown by the reduction

Figure 7 Immunostaining for JNK and p38 activation in NTN. (a) p-JNK immunostaining is absent in normal glomeruli. (b) Glomerular p-JNK staining is seen in capillary loops in 3-h vehicle rat NTN. (c) SYKi treatment abolished glomerular p-JNK in NTN. (d) Graph showing quantification of the number of p-JNK + cells per glomerular cross-section (gcs). (e) Some p-p38 + cells are seen in normal rat glomeruli. (f) Vehicle-treated rat (3 h) NTN showing an increase in p-p38 + cells. (g) SYKi treatment of NTN reduces glomerular p-p38 + cells. (h) Graph showing semi-quantitative scoring of glomerular p-p38 + cells. ${ }^{+++} P<0.001$ vs normal; ${ }^{* * *} P<0.001$ vs SYKi. 
in mRNA levels for individual pro-inflammatory mediators such as TNF- $\alpha$, blockade of which has been shown to suppress acute proteinuria in the first $24 \mathrm{~h}$ in this disease model. ${ }^{35}$ Of note, SYKi treatment also markedly suppressed expression of pro-inflammatory molecules at the $24 \mathrm{~h}$ time point in rat NTN when the neutrophil influx has largely
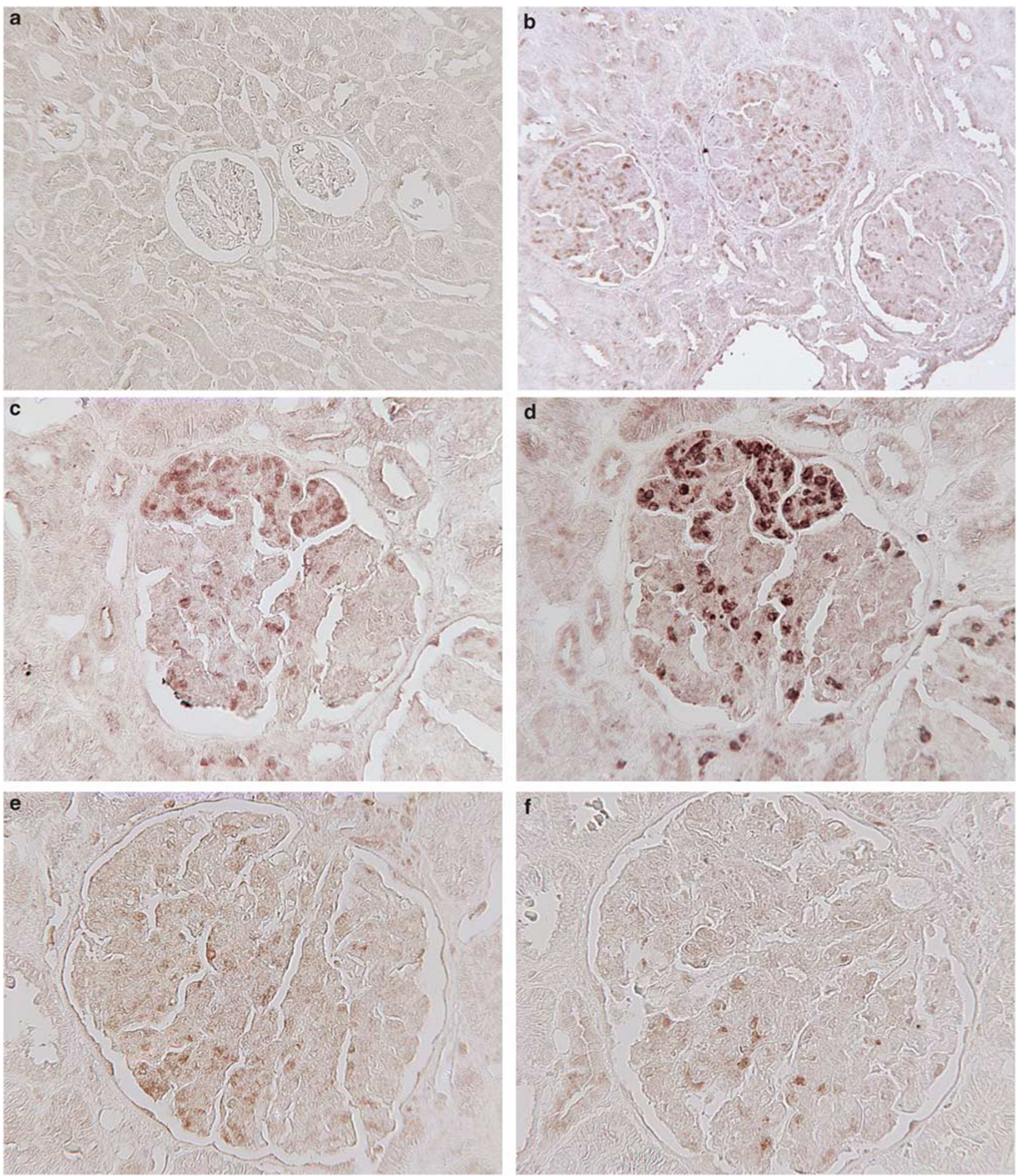

Figure 9 Syk activation in human kidney disease. (a) Minimal change disease has no immunostaining for p-Syk (Tyr 525/526). (b) A case of post-infectious glomerulonephritis shows p-Syk + cells in glomeruli. Serial sections in post-infectious glomerulonephritis shows a focal glomerular pattern of p-Syk + cells (c), which co-localises with neutrophils within capillary loops (d). In the same case, immunostaining shows the presence of (e) p-JNK + cells, and (f) $p$ p38 + cells, which have a similar glomerular distribution as that of p-Syk + cells. Original magnification, $\times 160(\mathbf{a}, \mathbf{b}), \times 400(\mathbf{c}-\mathbf{f})$. 
resolved and monocytes/macrophages are the main glomerular leukocyte population. Thus, it can be inferred that although SYKi treatment had only a minor effect upon macrophage accumulation at $24 \mathrm{~h}$, it had a profound inhibitory effect upon activation of the macrophage infiltrate, with suppression of macrophage-specific effector molecules such as NOS2 and MMP-12..$^{22,36,37}$ This is consistent with a previous study showing that a Syk inhibitor drug suppressed the macrophage-dependent phase of renal injury in NTN. ${ }^{18}$

Thirdly, an important mechanism through which SYKi treatment suppressed acute renal injury in rat NTN is the inhibition of p38 and JNK signalling. In vitro studies have linked $\mathrm{Fc}-\mathrm{R} \gamma$ activation to $\mathrm{p} 38$ and JNK signalling in mast cells, ${ }^{38}$ but there is little data to demonstrate such a Syk-p38/ JNK signalling pathway in vivo. In this study, we identified activation of Syk, p38 and JNK in infiltrating glomerular neutrophils in rat NTN, and demonstrated that SYKi treatment resulted in suppression of p38 and JNK activation. Furthermore, investigation of post-infectious glomerulonephritis identified glomerular activation of Syk, p38 and JNK, which exhibited similar immunostaining patterns, consistent with being activated in infiltrating neutrophils, although further work needs to be done to define Syk activation is a much wider range of human kidney diseases.

We have previously demonstrated that blockade of $\mathrm{p} 38 \alpha$ signalling significantly inhibits acute proteinuria in the first $24 \mathrm{~h}$ in this disease model. ${ }^{5}$ This protective effect was attributed to inhibition of glomerular platelet activation and neutrophil influx at the $3 \mathrm{~h}$ time point. Indeed, the inhibition of P-selectin immunostaining seen with SYKi treatment in the current study parallels that observed previously with p38 $\alpha$ blockade. ${ }^{5}$ JNK signalling also has a role in the induction of acute proteinuria in the first $24 \mathrm{~h}$ of rat NTN. Administration of a selective JNK inhibitor drug largely abrogated proteinuria in the first $24 \mathrm{~h}$ of rat NTN, although this did not affect the neutrophil influx at $3 \mathrm{~h} .{ }^{4}$ These studies, including direct addition of a JNK inhibitor to macrophages before adoptive transfer, have established that JNK signalling in macrophages is not essential for recruitment to the inflamed glomerulus, but that JNK signalling is required for activation of the macrophage pro-inflammatory response. ${ }^{4,22,39,40}$ The finding in the current study that SYKi treatment caused only a minor reduction in glomerular macrophages at $24 \mathrm{~h}$ in NTN, but yet markedly suppressed macrophage activation is consistent with the known effects of inhibition of JNK activation. Thus, the current study provides several lines of evidence to argue that Syk signalling is required for activation of the p38 and JNK pathways, and that this represents an important pathway of acute neutrophil-mediated glomerular injury.

One limitation in this type of study is the question of specificity of kinase inhibitor molecules. SYKi does have some activity against Flt-3, KDR and JAK-2 kinases, which could contribute to the efficacy of SYKi treatment, although SYKi has no activity against p38 or JNK. Ultimately, only a genetic approach using conditional deletion of the Syk gene can formally prove a pathogenic role of Syk in glomerulonephritis.

In summary, we have identified neutrophil Syk activation in acute experimental antibody-dependent glomerular injury and confirmed the relevance of this finding in human acute post-infectious glomerulonephritis. This study provides several lines of evidence to argue that Syk signalling is required for activation of the p38 and JNK pathways, and that this represents an important pathway of acute neutrophil-mediated glomerular injury in antibody-dependent kidney disease. These results identify Syk as a potential therapeutic target in antibody-dependent kidney disease such as the platelet and neutrophil-dependent glomerular injury in ANCA vasculitis.

\section{ACKNOWLEDGEMENTS}

This study was funded by the National Health and Medical Research Council of Australia and by Celgene.

\section{DISCLOSURE/CONFLICT OF INTEREST}

Celgene provided a portion of the funding of this study. MD and KB are employees of Celgene. DJN-P acts as a consultant for Celgene.

1. Nikolic-Paterson DJ, Atkins RC. The role of macrophages in glomerulonephritis. Nephrol Dial Transplant 2001;16:3-7.

2. Park SY, Ueda S, Ohno H, et al. Resistance of Fc receptor- deficient mice to fatal glomerulonephritis. J Clin Invest 1998;102:1229-1238.

3. Tang T, Rosenkranz A, Assmann KJ, et al. A role for Mac-1 (CDIlb/CD18) in immune complex-stimulated neutrophil function in vivo: Mac-1 deficiency abrogates sustained Fcgamma receptor-dependent neutrophil adhesion and complement-dependent proteinuria in acute glomerulonephritis. J Exp Med 1997;186:1853-1863.

4. Flanc RS, Ma FY, Tesch GH, et al. A pathogenic role for JNK signaling in experimental anti-GBM glomerulonephritis. Kidney Int 2007;72: 698-708.

5. Stambe C, Atkins RC, Tesch GH, et al. Blockade of p38alpha MAPK ameliorates acute inflammatory renal injury in rat anti-GBM glomerulonephritis. J Am Soc Nephrol 2003;14:338-351.

6. Yanagi S, Inatome R, Takano T, et al. Syk expression and novel function in a wide variety of tissues. Biochem Biophys Res Commun 2001;288:495-498.

7. Cheng AM, Rowley $B, P a o W$, et al. Syk tyrosine kinase required for mouse viability and B-cell development. Nature 1995;378:303-306.

8. Turner M, Mee PJ, Costello PS, et al. Perinatal lethality and blocked B-cell development in mice lacking the tyrosine kinase Syk. Nature 1995;378:298-302.

9. Costello PS, Turner M, Walters AE, et al. Critical role for the tyrosine kinase Syk in signalling through the high affinity lgE receptor of mast cells. Oncogene 1996;13:2595-2605.

10. Crowley MT, Costello PS, Fitzer-Attas CJ, et al. A critical role for Syk in signal transduction and phagocytosis mediated by Fcgamma receptors on macrophages. J Exp Med 1997;186:1027-1039.

11. Mocsai A, Ruland J, Tybulewicz VL. The SYK tyrosine kinase: a crucial player in diverse biological functions. Nat Rev Immunol 2010;10:387-402.

12. Genovese $M C$, Kavanaugh $A$, Weinblatt $M E$, et al. An oral syk kinase inhibitor in the treatment of rheumatoid arthritis: A 3 month randomized placebo controlled phase 2 study in patients with active RA who had failed biologic agents. Arthritis Rheum 2011;63:337-345.

13. Meltzer EO, Berkowitz RB, Grossbard EB. An intranasal Syk-kinase inhibitor (R112) improves the symptoms of seasonal allergic rhinitis in a park environment. J Allergy Clin Immunol 2005;115:791-796.

14. Weinblatt ME, Kavanaugh A, Burgos-Vargas $R$, et al. Treatment of rheumatoid arthritis with a Syk kinase inhibitor: a twelve-week, randomized, placebo-controlled trial. Arthritis Rheum 2008;58:3309-3318. 
15. Weinblatt $M E$, Kavanaugh A, Genovese $M C$, et al. An oral spleen tyrosine kinase (Syk) inhibitor for rheumatoid arthritis. N Engl J Med 2010;363:1303-1312.

16. Bahjat FR, Pine PR, Reitsma A, et al. An orally bioavailable spleen tyrosine kinase inhibitor delays disease progression and prolongs survival in murine lupus. Arthritis Rheum 2008;58:1433-1444.

17. Deng GM, Liu L, Bahjat FR, et al. Suppression of skin and kidney disease by inhibition of spleen tyrosine kinase in lupus-prone mice. Arthritis Rheum 2010;62:2086-2092.

18. Smith J, McDaid JP, Bhangal G, et al. A spleen tyrosine kinase inhibitor reduces the severity of established glomerulonephritis. J Am Soc Nephrol 2010;21:231-236.

19. Takazoe K, Tesch GH, Hill PA, et al. CD44-mediated neutrophil apoptosis in the rat. Kidney Int 2000;58:1920-1930.

20. Braselmann S, Taylor V, Zhao H, et al. R406, an orally available spleen tyrosine kinase inhibitor blocks fc receptor signaling and reduces immune complex-mediated inflammation. J Pharmacol Exp Ther 2006; 319:998-1008.

21. Lan HY, Mu W, Nikolic-Paterson DJ, et al. A novel, simple, reliable, and sensitive method for multiple immunoenzyme staining: use of microwave oven heating to block antibody crossreactivity and retrieve antigens. J Histochem Cytochem 1995;43:97-102.

22. Ma FY, Flanc RS, Tesch GH, et al. Blockade of the c-Jun amino terminal kinase prevents crescent formation and halts established anti-GBM glomerulonephritis in the rat. Lab Invest 2009;89: 470-484.

23. Ma FY, Tesch GH, Flavell RA, et al. MKK3-p38 signaling promotes apoptosis and the early inflammatory response in the obstructed mouse kidney. Am J Physiol Renal Physiol 2007;293:F1556-F1563.

24. Sada K, Takano T, Yanagi S, et al. Structure and function of Syk proteintyrosine kinase. J Biochem (Tokyo) 2001;130:177-186.

25. Lan HY, Paterson DJ, Atkins RC. Initiation and evolution of interstitial leukocytic infiltration in experimental glomerulonephritis. Kidney Int 1991;40:425-433.

26. Johnson RJ, Alpers CE, Pritzl $P$, et al. Platelets mediate neutrophildependent immune complex nephritis in the rat. J Clin Invest 1988; 82:1225-1235.

27. Feuerstein N, Chen F, Madaio M, et al. Induction of autoimmunity in a transgenic model of $B$ cell receptor peripheral tolerance: changes in coreceptors and B cell receptor-induced tyrosine-phosphoproteins. $\mathrm{J}$ Immunol 1999;163:5287-5297.
28. Kuligowski MP, Kitching AR, Hickey MJ. Leukocyte recruitment to the inflamed glomerulus: a critical role for platelet-derived P-selectin in the absence of rolling. J Immunol 2006;176:6991-6999.

29. Tipping $P G$, Huang $X R$, Berndt $M C$, et al. A role for $P$ selectin in complement-independent neutrophil-mediated glomerular injury. Kidney Int 1994;46:79-88.

30. Urzainqui A, Serrador JM, Viedma F, et al. ITAM-based interaction of ERM proteins with Syk mediates signaling by the leukocyte adhesion receptor PSGL-1. Immunity 2002;17:401-412.

31. Zarbock $A$, Abram CL, Hundt $M$, et al. PSGL-1 engagement by E-selectin signals through Src kinase Fgr and ITAM adapters DAP12 and FcR gamma to induce slow leukocyte rolling. J Exp Med 2008;205: 2339-2347.

32. Poole A, Gibbins JM, Turner M, et al. The Fc receptor gamma-chain and the tyrosine kinase Syk are essential for activation of mouse platelets by collagen. EMBO J 1997;16:2333-2341.

33. Mocsai $A$, Abram $C L$, Jakus $Z$, et al. Integrin signaling in neutrophils and macrophages uses adaptors containing immunoreceptor tyrosinebased activation motifs. Nat Immunol 2006;7:1326-1333.

34. Obergfell A, Eto K, Mocsai A, et al. Coordinate interactions of Csk, Src and Syk kinases with [alpha]llb[beta]3 initiate integrin signaling to the cytoskeleton. J Cell Biol 2002;157:265-275.

35. Lan HY, Yang N, Metz C, et al. TNF-alpha up-regulates renal MIF expression in rat crescentic glomerulonephritis. Mol Med 1997;3:136-144.

36. Bremer V, Tojo A, Kimura $\mathrm{K}$, et al. Role of nitric oxide in rat nephrotoxic nephritis: comparison between inducible and constitutive nitric oxide synthase. J Am Soc Nephrol 1997;8:1712-1721.

37. Tipping PG, Leong TW, Holdsworth SR. Tumor necrosis factor production by glomerular macrophages in anti-glomerular basement membrane glomerulonephritis in rabbits. Lab Invest 1991;65:272-279.

38. Okayama Y, Tkaczyk C, Metcalfe DD, et al. Comparison of Fc epsilon RIand Fc gamma RI-mediated degranulation and TNF-alpha synthesis in human mast cells: selective utilization of phosphatidylinositol-3-kinase for Fc gamma Rl-induced degranulation. Eur J Immunol 2003;33: 1450-1459.

39. Behmoaras J, Bhangal G, Smith J, et al. Jund is a determinant of macrophage activation and is associated with glomerulonephritis susceptibility. Nat Genet 2008;40:553-559.

40. Ikezumi $Y$, Hurst L, Atkins RC, et al. Macrophage-mediated renal injury is dependent on signaling via the JNK pathway. J Am Soc Nephrol 2004;15:1775-1784. 